\title{
BEAM TRANSMISSION AND EMITTANCE IN THE IPNS 50 MEV LINAC*
}

\author{
J. C. Dooling ${ }^{\dagger}$, F. R. Brumwell, L. I. Donley, G. E. McMichael, and V. F. Stipp \\ Argonne National Laboratory, Argonne, IL 60439, USA
}

\begin{abstract}
The Intense Pulse Neutron Source (IPNS) $50-\mathrm{MeV}$ Alvarez Drift-Tube Linac has been in operation since 1961 when it was commissioned as the injector for the Zero Gradient Synchrotron. In 1983, the original IPNS H' source was converted to a grooved-cathode, magnetron type providing reliable $40-50 \mathrm{~mA}$ operation at $30 \mathrm{~Hz}$. Transmission losses in the $750 \mathrm{kV}$ column, buncher, chopper, and transport line result in $25-30 \mathrm{~mA}$ of $\mathrm{H}^{-}$ injected into the linac. Typically, the average current out of the linac is 10-12 mA with an overall efficiency of 3540 percent. PARMILA studies along with a new energy and energy-spread diagnostic are being used to get a better understanding of emittance growth and beam-loss mechanisms. These studies examine the effect of injected energy, energy distribution and phase on the output beam in terms of transmission efficiency, energy, and energy spread. Effects of variations in the linac electromagnetic quadrupole gradients on transmission and emittance are assessed in a similar manner.
\end{abstract}

\section{LINAC INVESTIGATION}

The reasons for re-evaluating the LINAC with PARMILA are two-fold: In terms of total average current and beam losses, overall accelerator performance was not optimal. Data recorded with the Energy Spread and Energy Monitor[1] (ESEM) indicated that fluctuations were occurring in both the energy and energy spread of the beam out of linac. Modeling of the 450-MeV IPNS Rapid Cycling Synchrotron (RCS) suggested an input energy spread of at least $0.3 \mathrm{MeV}$ is required to damp instabilities before extraction. Also, initial tests with an early PARMILA model[2] were not successful in terms of setting quadrupole gradients within the DTL tank[3]. Since the PC version of both SUPERFISH and PARMILA are now available, a new attempt at modeling the linac has been undertaken.

\section{DESCRIPTION OF THE LINAC}

The IPNS $50 \mathrm{MeV}$ Alvarez Drift Tube Linac (DTL) was commissioned in 1961 as the injector for the Zero Gradient Synchrotron. Since that time, it has been in use almost continuously providing beam for a number of purposes, including isotope production for nuclear physics investigations and experiments for Ballistic Missile Defense. Since 1981, the linac has served as the injector for the IPNS RCS. The linac consists of a single copper-

\footnotetext{
*This work is supported by the USDOE:\#W-31-109-ENG-38 jicdooling@anl.gov
}

clad, steel tank, $33.5 \mathrm{~m}$ in length with an inner diameter of $95.1 \mathrm{~cm}$. The resonant frequency of the DTL tank is 200.07 MHz. Within the tank, 124 drift tubes house an equal number of independently-biased electromagnetic quadrupole magnets. The drift tubes were built in groups, but each one is essentially unique in its physical dimensions. Four different aperture diameters and six quadrupole lengths are used in the drift tubes. Variations in aperture and quadrupole lengths are given in Table 1.

Table 1: IPNS LINAC aperture and quadrupole data.

\begin{tabular}{|c|c|c|c|c|c|c|}
\hline Cell No. & $1-8$ & $\begin{array}{c}9- \\
18\end{array}$ & $\begin{array}{c}19- \\
33\end{array}$ & $\begin{array}{c}34- \\
58\end{array}$ & $\begin{array}{c}59- \\
83\end{array}$ & $\begin{array}{c}84- \\
124\end{array}$ \\
\hline Ap. ID $(\mathrm{cm})$ & 1.27 & 1.91 & 2.54 & 3.18 & 3.18 & 3.18 \\
\hline Quad L $(\mathrm{cm})$ & 3.02 & 4.45 & 6.03 & 8.73 & 14.29 & 19.05 \\
\hline
\end{tabular}

The first drift tube in the linac is embedded in the input endwall of the tank and forms a full cell with the next drift tube. At the high-energy end, a half-cell is formed between the last drift tube and the output endwall. The output endwall has no quadrupole.

\section{PARMILA MODELING}

Initial SUPERFISH modeling necessary for filling the SFDATA table has been done for six cells: 1, 23, 45, 71, 96 , and 124 . In the near future, a more refined model will be constructed looking at cells across the transitions described above, but the present set provides a useable baseline to begin examining the linac. The model begins with a transport section starting just upstream of the buncher. The emittance is estimated from old measurements made using a slit-scanner following the first set of triplet quadrupoles in the beamline between the source and linac. The measured normalized emittance, containing 90 percent of the beam, was $0.09 \pi$-cm-mrad in the horizontal plane and $0.14 \pi-\mathrm{cm}-\mathrm{mrad}$ in the vertical plane[4]. With a waist in both transverse planes at the center of the buncher cavity, Twiss parameters are estimated assuming a round beam cross section. The buncher aperture is $3.175 \mathrm{~cm}$ in diameter. The center of the buncher gap is $2.017 \mathrm{~m}$ from the input side of the first DTL quadrupole. Between the buncher cavity and DTL, a magnetic quadrupole triplet matches the beam into the acceptance of the DTL. The $750 \mathrm{keV}$ input beam has a $\beta=0.03994$; therefore, the $\beta \lambda$ product is $5.985 \mathrm{~cm}$ at the input. There are approximately $34 \beta \lambda$ periods between the center of the buncher gap and the first DTL quad. With input and output energies of $0.75 \mathrm{MeV}$ and $50 \mathrm{MeV}$, the unit cell length within the DTL varies from $6.0 \mathrm{~cm}$ to $47.4 \mathrm{~cm}$. 
The present modeling studies are on-going. To date, variations in beam phase and buncher voltage have been examined. Further studies will examine the effect of input energy, quadrupole gradient, and accelerating electric field fluctuations on transmission and energy spread. In the first set of studies, RF buncher voltage and phase relative to the main tank were varied. Figures 1 and 2 show the effects of these parameters on transmission and longitudinal emittance, respectively.

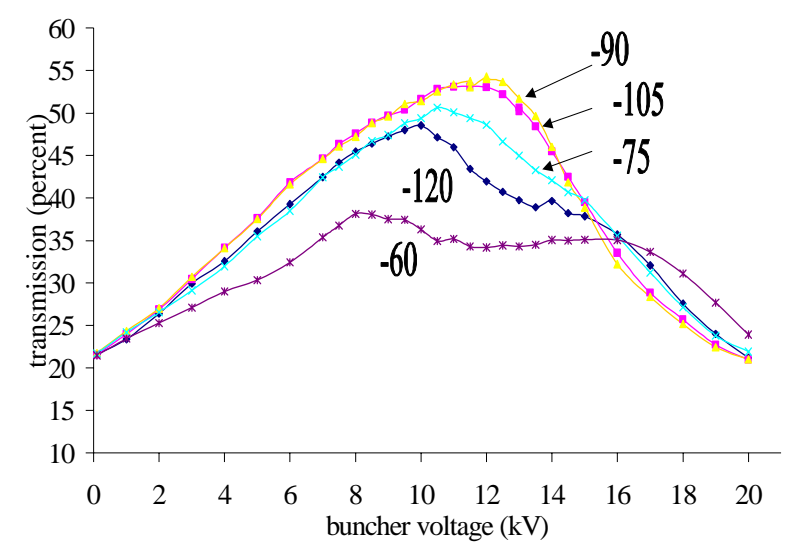

Figure 1: PARMILA LINAC transmission with buncher voltage and phase

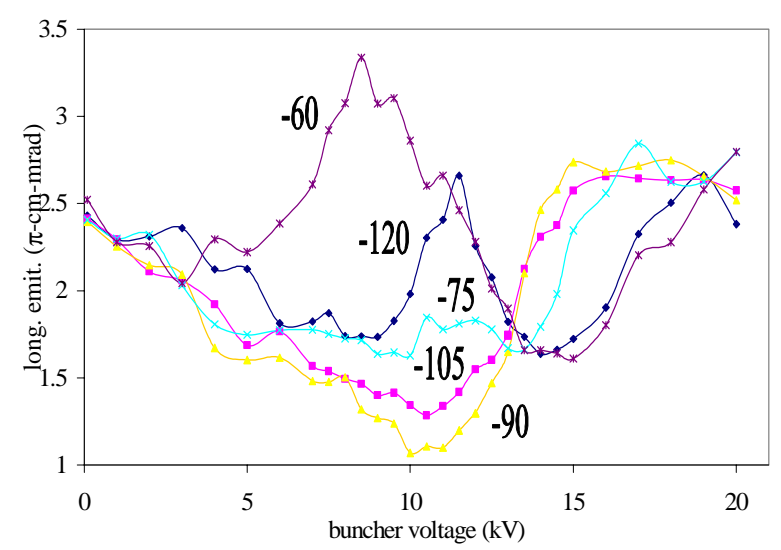

Figure 2: PARMILA LINAC output 90 percent longitudinal emittance with buncher voltage and phase.

\section{LINAC MEASUREMENTS}

A sudden change in the measured energy spread of the linac output beam occurring approximately 40-50 $\mu$ s into the linac macropulse has been observed persistently since August 2000. An ESEM record from the end of the May 2001 operations period is presented in Figure 3. The May 2001 run was characterized by greater instability and slightly reduced beam current $(<14 \mu \mathrm{A}$ versus $14.5-15.0$ $\mu \mathrm{A})$. Also, a dipole oscillation was observed in the RCS early in the acceleration cycle, $2 \mathrm{~ms}$ after injection. Measurements during the June 2001 run, show that the energy spread at the end of the macropulse has increased and the current has also increased to the 14.5-14.8 $\mathrm{mA}$ range; however, the dipole oscillation remains. Typical ESEM energy spread data from June is shown in Figure 4. June ESEM energy data is shown in Figure 5. Energy profiles are similar in May and June.

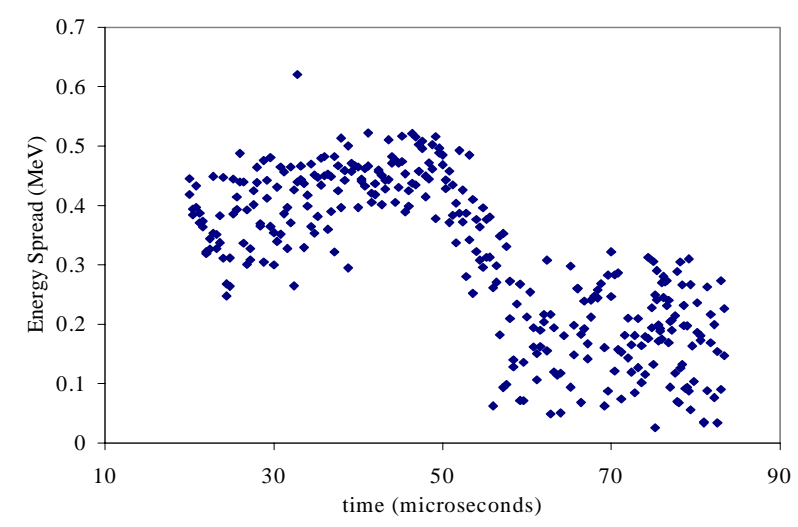

Figure 3: ESEM linac output energy spread, May 2001

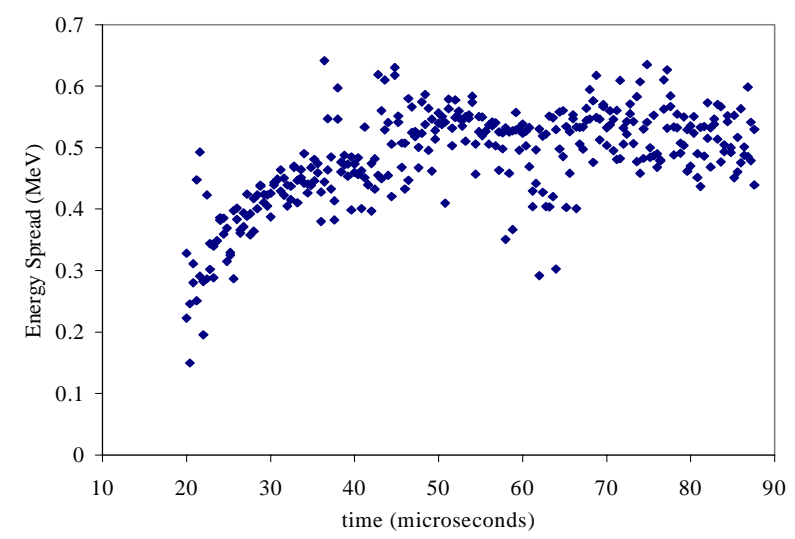

Figure 4: ESEM linac output energy spread ,June 2001

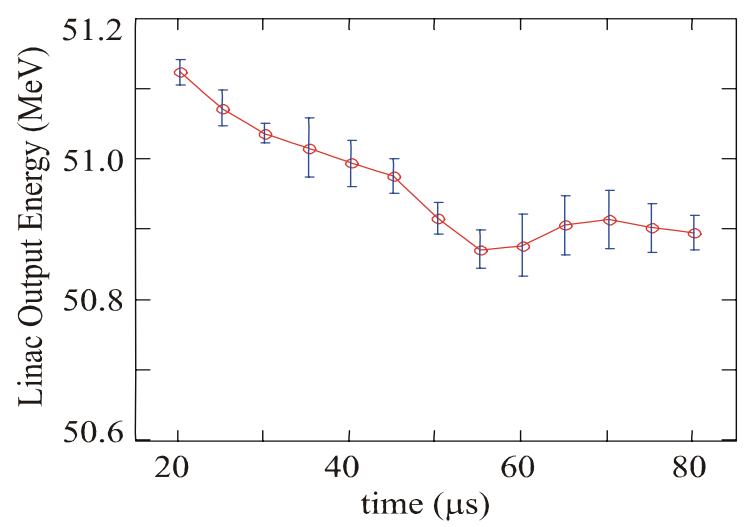

Figure 5: ESEM Linac output, June 2001

\section{DISCUSSION}

PARMILA indicates that average linac output energy is sensitive to the energy of the beam entering the buncher. Figure 5 presents PARMILA E-E $\mathrm{E}_{\mathrm{s}}$ output for a phase mismatch of $+20^{\circ}$ with respect to the linac rf. Relatively small changes in injected energy can lead to significant changes in the phase of the bunched beam relative to the rf in the DTL cavity. At $750 \mathrm{keV}$, the injected $\mathrm{H}^{-}$ions are 
essentially non-relativistic; therefore $\Delta \mathrm{W} / \mathrm{W} \approx 2 \Delta \mathrm{v} / \mathrm{v}$. Any error in input energy to the buncher will convert into a phase error at the entrance to the DTL multiplied by the number of $\beta \lambda$ periods between the buncher and linac. For example, a $1-\mathrm{keV}$ energy shift results in an $8^{\circ}$ phase shift with respect to the linac acceptance phase. This represents a variation of 0.13 percent in DC preaccelerator voltage. It is possible that the voltage may vary by as much as 0.5 percent during the macropulse ( 80 $\mu \mathrm{s})$. Figure 6 shows a significant longitudinal dipole oscillation in the linac resulting from an energy (phase) shift of $2.5 \mathrm{keV}\left(20^{\circ}\right)$

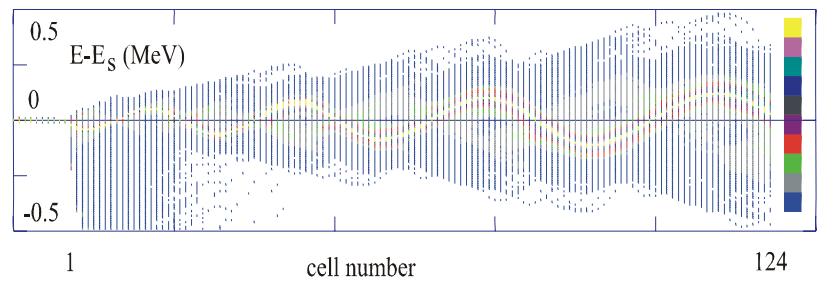

Figure 6: Linac energy spread for an input phase offset of $20^{\circ}$ with respect to the linac bucket, $\mathrm{V}_{\text {beff }}=8.5 \mathrm{kV}$.

PARMILA also predicts a rapid change in linac output emittance with buncher voltage that appears to be due to an increase in both energy spread and phase width of the microbunches. Longitudinal emittance is the product of energy spread and the phase width of the beam[5]: $\quad \varepsilon_{1}=\Delta \phi \Delta \mathrm{E}$. The energy distribution of the output bunch appears to undergo a bifurcation in energy, separating roughly into two equal-charge, high- and lowenergy lobes. The PARMILA longitudinal phase-space plot of the output bunch is presented in Figure 7. Overfocusing of the bunch at the entrance to the DTL seems to generate the observed energy splitting. In Figure 8, a simple buncher model shows the longitudinal density of the injected bunch for effective of buncher voltages of 10 and $13 \mathrm{kV}$. Also shown in the figure is the approximate acceptance phase. The synchronous phase, $\phi_{\mathrm{s}}=-25^{\circ}$, therefore the acceptance phase width $\approx\left|3 \phi_{\mathrm{s}}\right|=75^{\circ}$. Note that $75^{\circ} / 360^{\circ}=0.208$ or 20.8 percent, in good agreement with the predicted transmission efficiency for a DC beam shown in Figure 1.

PARMILA allows us to see how changes in injected energy, phase, and rf buncher voltage may impact bunch shape and phase at the DTL input, thereby modifying the energy and energy distribution out of the DTL. A connection has been made between these parameters and overall accelerator performance. A new buncher amplifier will help to better control rf phase and amplitude; however, the energy out of the pre-accelerator must also be better monitored.
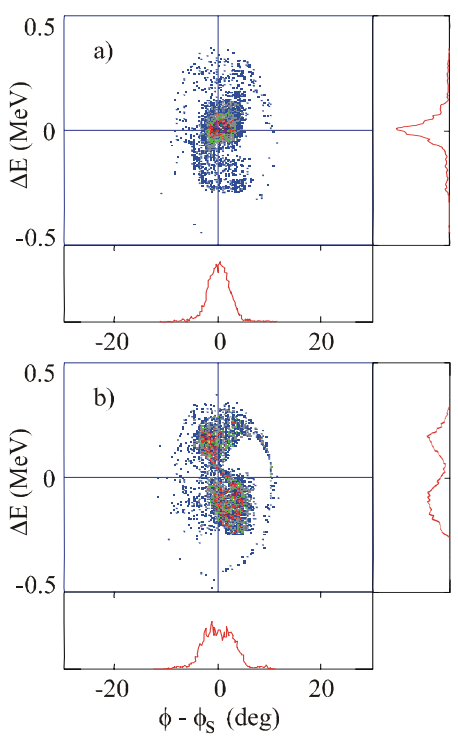

Figure 7: Final PARMILA energy and phase distribution for effective buncher voltages of a) $10 \mathrm{kV}$ and b) $13 \mathrm{kV}$.

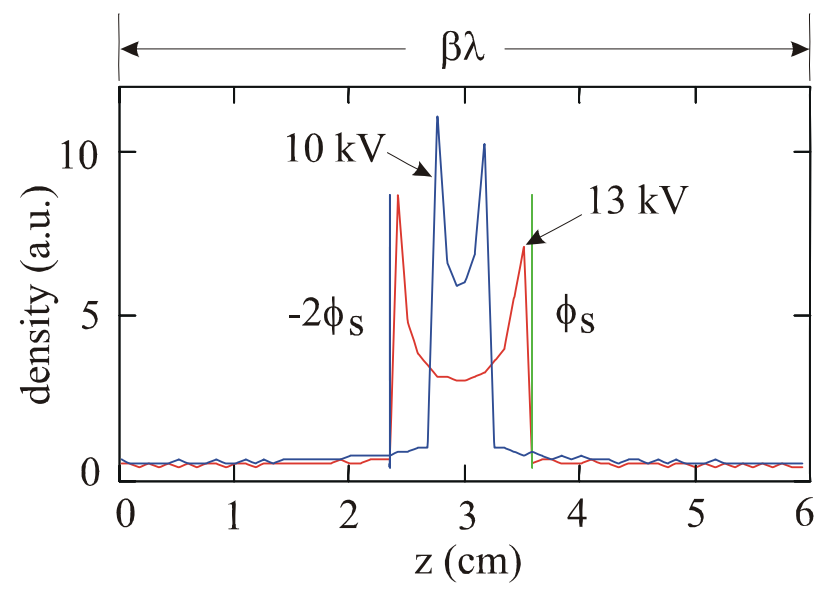

Figure 8: Simple buncher longitudinal density model at the first gap for buncher V: of $10 \mathrm{kV}$ and $13 \mathrm{kV}$. $\phi_{\mathrm{s}}=-25^{\circ}$.

\section{ACKNOWLEDGEMENTS}

This work is made possible by the consistent hard work of the IPNS Accelerator Operations Group.

\section{REFERENCES}

1. J.C. Dooling, et al., Proc. $20^{\text {th }}$ LINAC Conference, SLAC-R-561, Monterey, CA, August 2000, p 193.

2. B. Austin, et al. MURA Report 713, July 1, 1965.

3. V. Stipp, private communication.

4. V. Stipp, A. DeWitt, J. Madsen, IEEE Trans. Nucl. Sci., 30(4), 2743 (1983).

5. T. Wangler, $R F$ Linear Accelerators, Wiley, New York, 1998, p.264. 